TUBERCULOSIS

\title{
Empirical treatment with a fluoroquinolone delays the treatment for tuberculosis and is associated with a poor prognosis in endemic areas
}

\author{
J-Y Wang, P-R Hsueh, I-S Jan, L-N Lee, Y-S Liaw, P-C Yang, K-T Luh
}

Thorax 2006;61:903-908. doi: 10.1136/thx.2005.056887

See end of article for authors' affiliations

Correspondence to: Dr L-N Lee, Department of Laboratory Medicine, National Taiwan University Hospital, No 7, Chun Shan South Road, Taipei, 100, Taiwan; linalee@ccms.ntu. edu.tw

Received 3 December 2005 Accepted 9 June 2006

Published Online First

29 June 2006
Background: A study was conducted to evaluate the effect of the empirical use of fluoroquinolones on the timing of antituberculous treatment and the outcome of patients with tuberculosis in an endemic area. Methods: All patients with culture confirmed tuberculosis aged $\geqslant 14$ years diagnosed between July 2002 and December 2003 were included and their medical records were reviewed.

Results: Seventy nine (14.4\%) of the 548 tuberculosis patients identified received a fluoroquinolone (FQ group), 218 received a non-fluoroquinolone antibiotic ( $A B$ group), and 251 received no antibiotics before antituberculous treatment. Fifty two (65.8\%) experienced clinical improvement after fluoroquinolone use. In the $F Q$ group the median interval from the initial visit to starting antituberculous treatment was longer than in the $A B$ group and in those who received no antibiotics (41 $v 16 \vee 7$ days), and the prognosis was worse (hazard ratio $6.88(95 \% \mathrm{Cl} 1.84$ to 25.72$)$ ). More patients in the $\mathrm{FQ}$ and $A B$ groups were aged $>65$ years $(53.2 \%$ and $61.0 \%$ v $31.5 \%)$, had underlying disease $(53.2 \%$ and $46.8 \% \vee 34.3 \%)$, and were hypoalbuminaemic $(67.2 \%$ and $64.9 \% \vee 35.1 \%)$. Of the nine mycobacterial isolates obtained after fluoroquinolone use from nine patients whose initial isolates were susceptible to ofloxacin, one (11.1\%) was resistant to ofloxacin (after fluoroquinolone use for 7 days). Independent factors for a poor prognosis included empirical fluoroquinolone use, age $>65$, underlying disease, hypoalbuminaemia, and lack of early antituberculous treatment.

Conclusions: $14.4 \%$ of our patients with tuberculosis received a fluoroquinolone before the diagnosis. With a 34 day delay in antituberculous treatment and more frequent coexistence of underlying disease and hypoalbuminaemia, empirical fluoroquinolone treatment was associated with a poor outcome. Mycobacterium tuberculosis isolates could obtain ofloxacin resistance within 1 week.
$\mathrm{T}$ he fluoroquinolones (FQs) were introduced into clinical practice in the 1980s. With broad spectrum antimicrobial activity, they are recommended and widely used for the treatment of bacterial infections of the respiratory, gastrointestinal, and urinary tracts, as well as sexually transmitted diseases and chronic osteomyelitis. ${ }^{1-4}$ In contrast to many other antibiotics used to treat bacterial infections, the FQs have excellent in vitro and in vivo activity against Mycobacterium tuberculosis. ${ }^{56}$

A recent study conducted in United States showed that empirical FQ use was associated with a delay in the initiation of appropriate antituberculous (anti-TB) treatment. ${ }^{7}$ However, this important issue has not been addressed in an endemic area. It is also not clear whether the delay in anti-TB treatment affects survival. We therefore conducted a study to evaluate the impact of empirical use of FQs on the timing of anti-TB treatment and the outcome of patients with tuberculosis (TB) in an endemic area.

\section{METHODS}

This retrospective study was conducted in a 1500 bed tertiary care referral centre in northern Taiwan where the incidence and mortality of TB in 2003 was 62.4 and 5.8 per 100000 , respectively. ${ }^{8}$ We searched the records of the mycobacterial laboratory from July 2002 to December 2003 and found all the newly diagnosed patients aged $\geqslant 14$ years with culture confirmed TB. The medical records (including pharmacy records and the interview records from TB case managers) of each patient were reviewed. Acid-fast smear (AFS), mycobacterial culture, and susceptibility tests were performed as previously described. ${ }^{9}$ The chest radiographs were independently reviewed by two pulmonary specialists. If a discrepancy was noted between their interpretations, the image was further reviewed by one chest specialist blinded to the results.

Respiratory symptoms were defined as cough, dyspnoea, pleuritic chest pain, and haemoptysis. Constitutional symptoms included fever, malaise, night sweat, or weight loss. Headache or a change in consciousness were considered central nervous system symptoms. Musculoskeletal symptoms included arthralgia or backache. Abdominal symptoms were defined as abdominal pain or distention. Clinical improvement was defined as improvement of any of the presenting symptoms, chest radiographic findings, and laboratory data. ${ }^{7}$ Pulmonary TB confined below an imaginary line traced across the mid point of the hila and including the parahilar regions on the chest radiograph was defined as lower lung field TB. ${ }^{10}$ Disseminated TB was diagnosed if $M$ tuberculosis was isolated from the blood, bone marrow, liver biopsy specimen, or from two or more non-contiguous organs. ${ }^{11}$ Chronic renal failure was defined as a serum creatinine level higher than $20 \mathrm{mg} / \mathrm{l}^{10}{ }^{12}$

Standard anti-TB treatment consisted of isoniazid, rifampicin, ethambutol and pyrazinamide, and was modified according to the presence of concomitant hepatic and/or renal disease, adverse effects, and the results of drug susceptibility testing after it became available. In patients with liver disease pyrazinamide may be omitted. The anti-TB treatment was considered early if it was started within

Abbreviations: AFS, acid-fast smear; FQ, fluoroquinolone; TB, tuberculosis 
14 days of the initial visit and was judged as completed if fulfilling the definition of the World Health Organization (WHO). ${ }^{13}$ All patients were followed until they completed treatment or until 30 June 2005.

\section{Analysis of data}

Differences between groups were analysed using either an independent sample $t$ test or the Mann-Whitney $\mathrm{U}$ test for continuous variables and the $\chi^{2}$ test for categorical variables. Survival curves for each variable were generated using the Kaplan-Meier method and were compared using the log rank test. If a significant difference $(p<0.05)$ was found, the variables were entered into a multivariate survival analysis using Cox regression to identify factors independently associated with mortality.

\section{RESULTS}

Between July 2002 and December 2003 a total of 548 patients with newly diagnosed culture confirmed TB were identified. Pulmonary $\mathrm{TB}$ was diagnosed in 451 of them $(82.3 \%)$, including $38(6.9 \%)$ who had concomitant pulmonary and extrapulmonary TB. The remaining 97 patients $(17.7 \%)$ had only extrapulmonary involvement. Serological tests for HIV were performed in 296 patients and were positive in 17. Of the 252 patients with unknown HIV serostatus, all were free of other AIDS defined illnesses during follow up.

Seventy nine of the 548 patients $(14.4 \%)$ received a fluoroquinolone (FQ group) and 218 (39.8\%) received nonFQ antibiotics ( $\mathrm{AB}$ group) before the diagnosis of $\mathrm{TB}$. Antibiotics including FQs were prescribed more than once in 65 patients. The FQ prescribed was ciprofloxacin in 42 patients,

Table 1 Characteristics of the 548 patients with culture confirmed tuberculosis

\begin{tabular}{|c|c|c|c|}
\hline \multirow[b]{2}{*}{ Characteristic } & \multicolumn{3}{|c|}{ Empirical antibiotic use } \\
\hline & $\begin{array}{l}F Q \\
(n=79)\end{array}$ & $\begin{array}{l}\text { Others } \\
(n=218)\end{array}$ & $\begin{array}{l}\text { No antibiotic } \\
(n=251)\end{array}$ \\
\hline Age $>65$ years & $42(53.2 \%) \dagger$ & $133(61.0 \%) \ddagger$ & $79(31.5 \%)$ \\
\hline Male sex & 49 (62.0\%) & $150(68.8 \%)$ & $163(64.9 \%)$ \\
\hline Underlying disease & $42(53.2 \%) \dagger$ & $102(46.8 \%) \ddagger$ & $86(34.3 \%)$ \\
\hline Diabetes mellitus & $20(25.3 \%)$ & $51(23.4 \%)$ & $50(19.9 \%)$ \\
\hline Malignancy & $13(16.5 \%)$ & $29(13.3 \%)$ & $24(9.6 \%)$ \\
\hline Chronic renal insufficiency* & $8(10.1 \%)$ & $30(13.8 \%)$ & $7(2.8 \%)$ \\
\hline Receiving steroid & $5(6.3 \%)$ & $6(2.8 \%)$ & $11(4.4 \%)$ \\
\hline AIDS & $5(6.3 \%)$ & $10(4.6 \%)$ & $5(2.0 \%)$ \\
\hline Liver cirrhosis & $2(2.5 \%)$ & $5(2.3 \%)$ & $6(2.4 \%)$ \\
\hline Alcoholism & 0 & $1(0.5 \%)$ & $3(1.2 \%)$ \\
\hline \multicolumn{4}{|l|}{ Smoking } \\
\hline Current smoker & $17(21.5 \%)$ & $62(28.4 \%)$ & $72(28.3 \%)$ \\
\hline Ex-smoker & $14(17.7 \%)$ & $27(12.4 \%)$ & $25(10.0 \%)$ \\
\hline Extrapulmonary TB & $20(25.3 \%)$ & $72(33.0 \%) \ddagger$ & $43(17.1 \%)$ \\
\hline TB pleurisy or peritonitis & $11(13.9 \%)$ & $43(19.7 \%)$ & $22(8.8 \%)$ \\
\hline Musculoskeletal TB & $4(5.1 \%)$ & $9(4.1 \%)$ & $7(2.8 \%)$ \\
\hline TB lymphadenopathy & $0(0 \%)$ & $6(2.8 \%)$ & $8(3.2 \%)$ \\
\hline Urogenital TB & $1(1.3 \%)$ & $10(4.6 \%)$ & $3(1.2 \%)$ \\
\hline TB meningitis & $1(1.3 \%)$ & $1(0.5 \%)$ & $3(1.2 \%)$ \\
\hline Disseminated TB & $3(3.8 \%)$ & $3(1.4 \%)$ & 0 \\
\hline \multicolumn{4}{|l|}{ Symptom at presentation } \\
\hline Respiratory symptoms & $52(65.8 \%)$ & $127(58.3 \%) \ddagger$ & $177(70.5 \%)$ \\
\hline Constitutional symptoms & $41(51.9 \%) \dagger$ & $103(47.2 \%) \ddagger$ & $60(23.9 \%)$ \\
\hline CNS symptoms & $4(5.1 \%)$ & $14(6.4 \%)$ & $7(2.8 \%)$ \\
\hline Neck mass & 0 & $5(2.3 \%)$ & $6(2.4 \%)$ \\
\hline Dysuria & 0 & $3(1.4 \%)$ & $2(0.8 \%)$ \\
\hline Musculoskeletal symptoms & $2(2.5 \%)$ & $5(2.3 \%)$ & $6(2.4 \%)$ \\
\hline Abdominal symptoms & $2(2.5 \%)$ & $10(4.6 \%)$ & $8(3.2 \%)$ \\
\hline None (incidental finding) & $4(5.1 \%)$ & $17(7.8 \%)$ & $29(11.6 \%)$ \\
\hline Symptom $>14$ days & $46(58.2 \%) \dagger$ & $122(56.0 \%) \ddagger$ & $193(76.9 \%)$ \\
\hline Ordering CXR at initial visit in PTB & $69(87.3 \%)$ & $174(79.8 \%)$ & $194(77.3 \%)$ \\
\hline \multicolumn{4}{|l|}{ CXR finding } \\
\hline \multicolumn{4}{|l|}{ Pattern } \\
\hline Fibronodular infiltrates & $25(31.6 \%)$ & $66(30.3 \%)$ & $84(33.5 \%)$ \\
\hline Alveolar consolidation & $33(41.8 \%)$ & $86(39.4 \%)$ & $77(30.7 \%)$ \\
\hline Multiple nodules or mass & $9(11.4 \%)$ & $20(9.2 \%)$ & $44(17.5 \%)$ \\
\hline Fibrotic change & $5(6.3 \%)$ & $15(6.9 \%)$ & $12(4.8 \%)$ \\
\hline Miliary shadowing & $1(1.3 \%)$ & $8(3.7 \%)$ & $10(4.0 \%)$ \\
\hline No parenchymal lesion & $6(7.6 \%)$ & $23(10.6 \%)$ & $24(9.6 \%)$ \\
\hline Lower lung field TB & $18(22.8 \%)$ & $47(21.6 \%) \ddagger$ & $36(14.3 \%)$ \\
\hline Cavity & $9(11.4 \%)$ & $21(9.6 \%) \ddagger$ & $43(17.1 \%)$ \\
\hline \multicolumn{4}{|l|}{ Albumin $(\mathrm{g} / \mathrm{dl})$} \\
\hline$<35 / \geqslant 35$ & $43(67.2 \%) / 21 \dagger$ & $113(64.9 \%) / 61 \ddagger$ & $54(35.1 \%) / 100$ \\
\hline Mean (SD) & $31.7(6.2) \dagger$ & $32.2(6.1) \ddagger$ & $37.4(6.4)$ \\
\hline Sputum AFS+ in PTB & $8(12.1 \%)+$ & $53(24.3 \%)$ & $65(25.9 \%)$ \\
\hline Single drug resistance & $20(25.3 \%)$ & $48(22.0 \%)$ & $73(29.1 \%)$ \\
\hline Multidrug resistance & $10(12.7 \%)$ & $17(7.8 \%)$ & $29(11.6 \%)$ \\
\hline \multicolumn{4}{|c|}{$\begin{array}{l}\text { AFS, acid fast smear; AIDS, acquired immunodeficiency syndrome; CNS, central nervous system; CXR, ches } \\
\text { radiograph; PTB, pulmonary tuberculosis; TB, tuberculosis. } \\
{ }^{*} \text { Chronic renal insufficiency was defined as a serum creatinine level }>20 \mathrm{mg} / \mathrm{l} .{ }^{12} \\
\mathrm{tp}<0.05 \mathrm{FQ} \text { group } v \text { no antibiotic group. } \\
\mathrm{tp}<0.05 \text { other antibiotic group } v \text { no antibiotic group. } \\
\mathrm{Tp}<0.05 \mathrm{FQ} \text { group } v \text { other antibiotic group. }\end{array}$} \\
\hline
\end{tabular}




\begin{tabular}{|c|c|c|c|c|}
\hline \multirow[b]{2}{*}{ Characteristic } & \multicolumn{4}{|c|}{ Empirical antibiotic use } \\
\hline & $\begin{array}{l}\mathrm{FQ} \\
(\mathrm{n}=79)\end{array}$ & $\begin{array}{l}F Q, n o \text { underlying } \\
\text { disease } \\
(n=37)\end{array}$ & $\begin{array}{l}\text { Others } \\
\text { ( } n=218 \text { ) }\end{array}$ & $\begin{array}{l}\text { No } \\
(n=251)\end{array}$ \\
\hline $\begin{array}{l}\text { Initial visit to ordering TB studies } \\
\text { (days) }\end{array}$ & $6(0-173)+\pi$ & $6(1-173)$ & $5(0-163)$ & $5(0-272)$ \\
\hline AFS $+(n=126)$ & $9(1-173)$ & $4(1-173)$ & $3(0-78)$ & $3(0-112)$ \\
\hline AFS $-(n=422)$ & $6(0-159)$ & $6(1-61)$ & $5(0-163)$ & $5(0-272)$ \\
\hline TB study to anti-TB treatment (days) ${ }^{*}$ & $25(-3-231)+\bullet$ & $31(-3-73)$ & $7(-35-143) \ddagger$ & $0(-114-146)$ \\
\hline AFS+ $(n=123)$ & $5(1-21) \dagger$ & $4(2-9)$ & $2(-16-98) \ddagger$ & $-1(-35-55)$ \\
\hline AFS $-(n=363)$ & $31(-3-231)+\uparrow$ & $33(-3-73)$ & $16(-35-143) \neq$ & $1(-114-146)$ \\
\hline Initial visit to anti-TB treatment (days) & $41(6-233)+\bullet$ & $38(6-175)$ & $16(0-198) \pm$ & $7(0-286)$ \\
\hline$A F S+(n=123)$ & $15(8-175)+\overbrace{}^{\bullet}$ & $10(8-175)$ & $6(0-105) \ddagger$ & $3(0-123)$ \\
\hline AFS- $(n=363)$ & $42(6-233)+4$ & $39(6-96)$ & $27(0-198) \ddagger$ & $13(0-286)$ \\
\hline Ordering TB study at initial visit & $53(67.1 \%)$ & $26(70.3 \%)$ & $163(74.8 \%)$ & $179(71.3 \%)$ \\
\hline $\begin{array}{l}\text { TB study to identification available } \\
{\text { (days) }{ }^{*}}\end{array}$ & $63(37-85)$ & $64(55-82)$ & $62(36-92)$ & $62(35-121)$ \\
\hline AFS $+(n=126)$ & $63(57-73)$ & $60(57-62)$ & $63(36-78)$ & $63(50-77)$ \\
\hline AFS $-(n=422)$ & $63(37-85)$ & $64(55-82)$ & $62(54-92)$ & $62(35-121)$ \\
\hline Outcome & 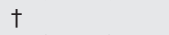 & & & \\
\hline Completed treatment & 41 (51.9\%) & $23(62.2 \%)$ & $133(61.0 \%)$ & 198 (78.9\%) \\
\hline Ongoing treatment & 0 & 0 & $1(0.5 \%)$ & $4(1.6 \%)$ \\
\hline Not treated & $2(2.5 \%)$ & $2(5.4 \%)$ & $5(2.3 \%)$ & $1(0.4 \%)$ \\
\hline Lost to follow up & $13(16.5 \%)$ & $7(18.9 \%)$ & $34(15.6 \%)$ & $33(13.1 \%)$ \\
\hline Mortality & $23(29.1 \%)$ & $5(13.5 \%)$ & $45(20.6 \%)$ & $15(6.0 \%)$ \\
\hline Died of TB & 12 & 3 & 22 & 1 \\
\hline Died with TB & 11 & 2 & 23 & 14 \\
\hline Length of survival (days)* & $85.5(18-448) \dagger$ & $59(26-448)$ & 55 (1-708)‡ & $249(23-902)$ \\
\hline \multicolumn{5}{|c|}{$\begin{array}{l}\text { AFS, acid-fast smear; } T B \text {, tuberculosis. } \\
\text { *Data presented as median (range). } \\
t p<0.05 \mathrm{FQ} \text { group } v \text { no antibiotic group. } \\
\pm \mathrm{f}<0.05 \text { other antibiotic group } v \text { no antibiotic group. } \\
\uparrow \mathrm{p}<0.05 \mathrm{FQ} \text { group } v \text { other antibiotic group. }\end{array}$} \\
\hline
\end{tabular}

levofloxacin in 21, and moxifloxacin in the remaining 16, and the mean (SD) duration of use was 9.5 (6.0) days. The initial diagnosis was community acquired pneumonia in 69 patients $(87.3 \%)$, septic arthritis in four $(5.1 \%)$, bacterial peritonitis in three $(3.8 \%)$, and one $(1.3 \%)$ each for urinary tract infection, meningitis, and fever of unknown origin.

In 18 patients $M$ tuberculosis isolates before and after the use of FQs were preserved. Half of them had clinical specimens collected $\geqslant 7$ days after the use of FQs (range 7-18 days). All of the 36 isolates were susceptible to ofloxacin, except one from a patient after using a FQ for 7 days. The patient presented with right knee arthralgia for 1 week and had not taken any medication before coming to our hospital. Among the 469 patients without empirical FQ use, 177 had previously been treated by local doctors and 76 of them had received antibiotics (non-FQ antibiotics in 52, unknown in the remainder). Of the other 292 patients, 137 were not likely to have been treated owing to the short duration of symptoms $(\leqslant 14$ days $)$.

The clinical characteristics of the 548 patients are summarised in table 1 . The age, presence of underlying disease, extrapulmonary involvement, respiratory or constitutional symptoms, duration of symptoms, pulmonary cavitation, serum albumin level, and result of sputum AFS were significantly different in the three groups. Of the 50 asymptomatic patients, $24(48 \%)$ had only one positive mycobacterial culture; 23 of these had a pulmonary lesion which resolved after anti-TB treatment and only one $(0.2 \%)$ with TB peritonitis had normal chest images.

Anti-TB treatment was started within 14 days of the initial visit in 249 patients $(45.4 \%)$, including nine $(11.4 \%)$ in the FQ group, 90 (41.3\%) in the AB group, and 150 (59.8\%) in the control group. In comparison with patients in the other two groups, for those in the FQ group there was a delay in testing $(p=0.037$ for non-FQ antibiotic group; $p=0.040$ for no antibiotic group) and treatment for TB $(p<0.001$ for both groups), and patients in this group had a worse prognosis
( $p<0.001$ for no antibiotic group) (table 2). For patients with smear negative specimens, those in the FQ group were treated a median of 42 days after the initial visit and 31 days after mycobacterial culture was ordered while, in the other two groups, patients were treated 27 and 13 days after the initial visit and only 16 and 1 days after mycobacterial culture was requested, respectively $(\mathrm{p}<0.001$ for both groups). Fifty two patients in the FQ group (65.8\%) experienced clinical improvement in symptoms $(n=40)$, chest radiographic findings $(n=19)$, or laboratory data $(n=14)$ in a mean of 4 days after empirical use of FQs (range 1-14 days).

Of the 12 patients in the FQ group who died of TB (table 2), the cause of death was septic shock without any bacteriological evidence other than $M$ tuberculosis infection in eight, respiratory failure due to extensive pulmonary inflammation in three, and sputum impaction in one. No events of sudden death as a result of prolongation of the corrected QT interval (QTc) were noted. In the other two groups, 15 patients died of septic shock without any evidence of concomitant bacterial infection other than $M$ tuberculosis and eight died of respiratory failure (seven due to severe caseous pneumonia and one to sputum impaction). A total of 45 patients ( 13 in the FQ group, 30 in the $\mathrm{AB}$ group, and two in the control group) died within 3 months of the initial visit. At the terminal stage ciprofloxacin resistant bacteria were isolated from eight $(61.5 \%)$, eight $(26.7 \%)$, and one $(50.0 \%)$ of the three groups, respectively $(\mathrm{p}=0.090)$. Among these, multidrug resistant Pseudomonas aeruginosa or Acinetobacter baumannii, defined as resistance to two or more classes of antipseudomonal agents, ${ }^{14}$ were isolated from four, two, and one, respectively $(p=0.052)$. One patient in the FQ group, two in the non-FQ antibiotic group, and five in the no antibiotic group died after completion of TB treatment.

To identify the factors associated with the different prognosis in the three groups, survival analysis was performed on the 11 variables with significant inter-group 


\begin{tabular}{|c|c|c|c|c|}
\hline & \multirow{2}{*}{$\begin{array}{l}\text { No of } \\
\text { patients }\end{array}$} & \multirow[b]{2}{*}{ Mortality (\%) } & \multicolumn{2}{|l|}{ Hazard ratio } \\
\hline & & & Univariate analysis & Multivariate analysis* \\
\hline \multicolumn{5}{|l|}{ Empirical antibiotic use } \\
\hline Fluoroquinolone & 37 & $5(13.5 \%)$ & 6.88 (1.84 to 25.72$)$ & 4.22 (1.01 to 11.82$)$ \\
\hline Others & 116 & $14(12.1 \%)$ & $5.19(1.71$ to 15.79$)$ & $1.36(0.33$ to 5.56$)$ \\
\hline No & 165 & $4(2.4 \%)$ & & \\
\hline \multicolumn{5}{|l|}{ Age } \\
\hline$>65$ years & 131 & $19(14.5 \%)$ & 7.08 (2.41 to 20.81$)$ & $6.43(1.37$ to 30.29$)$ \\
\hline$\leqslant 65$ years & 187 & $4(2.1 \%)$ & & \\
\hline \multicolumn{5}{|l|}{ Involvement of TB } \\
\hline Extrapulmonary & 81 & $8(9.9 \%)$ & $1.38(0.58$ to 3.26$)$ & \\
\hline Pulmonary & 237 & $15(6.3 \%)$ & & \\
\hline \multicolumn{5}{|l|}{ Respiratory symptoms } \\
\hline Yes & 224 & $10(4.5 \%)$ & 0.34 (0.15 to 0.77$)$ & $0.66(0.20$ to 2.16$)$ \\
\hline No & 94 & $13(13.8 \%)$ & & \\
\hline \multicolumn{5}{|l|}{ Constitutional symptoms } \\
\hline Yes & 105 & $12(11.4 \%)$ & $2.25(0.99$ to 5.10$)$ & \\
\hline No & 213 & $11(5.2 \%)$ & & \\
\hline \multicolumn{5}{|l|}{ Duration of symptoms } \\
\hline$>14$ days & 217 & $10(4.6 \%)$ & $0.31(0.13$ to 0.70$)$ & $0.74(0.26$ to 2.10$)$ \\
\hline$\leqslant 14$ days & 101 & $13(12.9 \%)$ & & \\
\hline \multicolumn{5}{|l|}{ Lower lung field TB } \\
\hline Yes & 61 & $4(6.6 \%)$ & $0.89(0.30$ to 2.62$)$ & \\
\hline No & 257 & $19(7.4 \%)$ & & \\
\hline \multicolumn{5}{|l|}{ Cavity on chest radiograph } \\
\hline Yes & 42 & $3(7.1 \%)$ & $1.03(0.31$ to 3.47$)$ & \\
\hline No & 276 & $20(7.2 \%)$ & & \\
\hline \multicolumn{5}{|l|}{ Albumin (g/dl) } \\
\hline$<35$ & 76 & 15 (19.7\%) & 15.07 (3.44 to 66.05$)$ & 8.85 (1.96 to 39.94$)$ \\
\hline$\geqslant 35$ & 119 & $2(1.7 \%)$ & & \\
\hline \multicolumn{5}{|l|}{ Sputum acid-fast smear } \\
\hline Positive & 61 & $2(3.3 \%)$ & 0.38 (0.09 to 1.64$)$ & \\
\hline Negative & 257 & $21(8.2 \%)$ & & \\
\hline \multicolumn{5}{|l|}{ Anti-TB treatment } \\
\hline$\leqslant 14$ days after initial visit & 162 & $7(4.3 \%)$ & $0.37(0.15$ to 0.91$)$ & $0.70(0.51$ to 0.97$)$ \\
\hline$>14$ days after initial visit & 156 & $16(10.3 \%)$ & & \\
\hline
\end{tabular}

Data shown are hazard ratios with $95 \%$ confidence intervals (CI).

*Multivariate survival analysis was performed for the 195 patients with complete data.

differences as well as the factor "empirical antibiotic use" itself. Multivariate survival analysis for the 548 patients showed that the poor prognostic factors included empirical FQ use (hazard ratio (HR) 2.39 (95\% CI 1.20 to 4.76$)$ ), age $>65$ years (HR 4.13 (95\% CI 2.3 to 7.42$)$ ), presence of underlying disease (HR 2.79 (95\% CI 1.61 to 4.84)), serum albumin level $<3.5 \mathrm{~g} / \mathrm{dl}$ (HR 3.14 (95\% CI 1.73 to 5.71 )), and lack of anti-TB treatment within 14 days of the initial visit (HR 2.04 (95\% CI 1.18 to 3.57)). Because a patient with underlying disease is more likely to have a low serum albumin level and empirical treatment with an FQ is more likely for an acute illness, we excluded this subgroup and analysed the effect on prognosis of empirical antibiotic use in the 318 patients without underlying disease (table 3 ). From table 2 it appears that empirical antibiotic use (including FQs and non-FQ antibiotics) is associated with increased mortality in univariate analysis but that, after adjustment for confounders, the effect of non-FQ antibiotics is not significantly different from no antibiotic treatment whereas the increased mortality with FQs remains strong and significant. Other independent factors for a poor prognosis included age $>65$ years, hypoalbuminaemia, and lack of early anti-TB treatment.

\section{DISCUSSION}

Our findings have confirmed that the empirical use of antibiotics, especially FQs, for presumed bacterial infection could delay the treatment for TB, especially in patients with smear negative specimens. ${ }^{75-18}$ Our results also show that patients who receive an FQ (but not non-FQ antibiotics) before standard anti-TB treatment have a poorer prognosis, most likely as a result of the emergence of drug resistant bacteria and the association with ageing, underlying disease, malnutrition, and delayed treatment. With empirical use of FQ for $1-3$ weeks, $11.1 \%$ of the $M$ tuberculosis isolates became ofloxacin resistant.

In a study conducted in Baltimore where the incidence of $\mathrm{TB}$ is relatively low, empirical FQ treatment was given to 16 patients with TB during a 40 month period and was associated with a significant delay in starting anti-TB treatment ( $21 \quad v \quad 5$ days $).^{7}$ Although the proportion of TB patients who received FQs in our study was less than that in Baltimore $(14.4 \% \vee 48 \%$ ), we found that the impact of widespread FQ use was greater in an endemic area of TB, with 79 patients during an 18 month period receiving empirical FQ treatment in whom anti-TB treatment was significantly delayed (table 2). Most of this delay occurred between the time that the bacteriological tests were ordered and the anti-TB treatment was started. However, the time required for the mycobacterial isolates to grow was similar for the three groups.

The proportion of patients who received TB tests at the initial visit was not significantly different, but the time from the initial visit to ordering TB tests was significantly longer in the FQ group. There are two possible reasons for these findings. Firstly, with the excellent in vivo activity of FQs against $M$ tuberculosis, ${ }^{5}{ }^{6}$ about two thirds of our patients with TB who had received an FQ showed clinical improvement so laboratory tests for TB were often not ordered until a later date (17.5 days $v 11.4$ days); this difference was not statistically significant. Even if the laboratory tests were performed with a partly improved clinical course and low index of suspicion, anti-TB treatment would not be started until the culture became positive and $M$ tuberculosis was identified. Secondly, more patients who received an FQ were 
elderly and had hypoalbuminaemia and underlying comorbid conditions, implying a poor general condition. They might therefore be too weak to expectorate adequate sputum specimens for TB tests which could result in a low smear positive rate and delayed treatment.

Our analysis showed that the empirical use of FQ was independently associated with a poor prognosis, most probably owing to the emergence of ciprofloxacin resistant isolates and multidrug resistant $P$ aeruginosa or $A$ baumannii, which has been reported to increase infection related mortality and a deterioration in the survival rate. ${ }^{14}{ }^{19}$ In addition, inappropriate use of FQs can result in the development of FQ resistance in $M$ tuberculosis. ${ }^{20-22}$ Although we were unable to verify the previous medication history, our finding was consistent with the case reported by Ginsburg et al who showed that FQ resistance can develop after only 13 days of exposure and suggested that mycobacterial resistance to FQ could potentially occur during a short course of treatment for a common bacterial infection. The delay in anti-TB treatment resulting from empirical FQ use further increased the morbidity and reduced the survival of TB patients. ${ }^{23-25}$ In agreement with our previous study, ${ }^{10}$ the survival analysis showed that death from TB was significantly affected by the presence of conditions that would alter cell mediated immunity including ageing, ${ }^{26}$ underlying systemic diseases such as diabetes mellitus, ${ }^{27}$ malignancy, ${ }^{28}$ and chronic renal insufficiency, ${ }^{29}$ and a low serum albumin level, ${ }^{30}$ further emphasising the importance of cell mediated immunity in the defence from TB infection.

We also found that the empirical use of non-FQ antibiotics was associated with a delay in anti-TB treatment but not in ordering TB tests, which suggests that the delay was due to the time inherent in taking a course of antibiotics and waiting to see if there is a clinical response. ${ }^{18}$ Like patients in the FQ group, those in the non-FQ antibiotic group were older and more poorly nourished, more often had underlying diseases, and presented acutely with constitutional symptoms and lower lobe infiltrates (table 1) than those who had received no previous antibiotics. They therefore tended to have an initial diagnosis of acute pneumonia which contributed to the delay in anti-TB treatment. However, the delay in this group was less than that in the FQ group, probably because the progressively deteriorating disease raised the suspicion of TB and prompted anti-TB treatment. Patients in the FQ group, on the other hand, could show improvement with the use of FQ treatment which masked the presence of TB and markedly delayed the diagnosis. Although patients in the FQ and non-FQ antibiotic groups were similar in age, degree of malnourishment and underlying disease, those in the non-FQ group who died tended to die earlier than in the FQ group (table 2), probably because the bactericidal effect of the FQ on $M$ tuberculosis partially alleviated the progression and prevented early mortality.

Our study was limited by the possible bias in patient selection because it was conducted in a tertiary care referral centre and only culture confirmed cases of TB were included. Another major limitation was the unavailability of the previous medication history, as many of the patients not treated empirically with FQs could have received FQs before coming to our hospital. However, our analysis showed that the widespread use of FQs for the treatment of bacterial infection resulted in a delay in the treatment of $\mathrm{TB}$ and a worse outcome in an endemic area. Our data revealed that $14.4 \%$ of our TB patients received FQs before diagnosis of TB. By causing the emergence of resistant bacteria, a significant delay in the initiation of anti-TB treatment, and association with conditions that compromise cellular immunity, the empirical use of FQs in patients with TB was associated with a poor outcome. In addition, the $M$ tuberculosis isolates could become resistant to ofloxacin within 1 week. We therefore conclude that, in an endemic area of TB, a high index of suspicion is required. $M$ tuberculosis should be considered as a possible causative pathogen in every infectious disease, whether or not clinical improvement is noted after the use of an FQ, and appropriate bacteriological and histopathological tests for TB should be performed as early as possible.

\section{Authors' affiliations}

J-Y Wang, Y-S Liaw, P-C Yang, Department of Internal Medicine, National Taiwan University Hospital, Taipei, Taiwan

P-R Hsueh, I-S Jan, L-N Lee, K-T Luh, Laboratory Medicine, National Taiwan University Hospital, Taipei, Taiwan

Funding: none.

Competing interests: none declared.

\section{REFERENCES}

1 Bartlett JG, Dowell SF, Mandell LA, et al. Practice guidelines for the management of community-acquired pneumonia in adults. Infectious Diseases Society of America. Clin Infect Dis 2000:31:347-82

2 Neu HC. Clinical use of the quinolones. Lancet 1987;2:1319-22.

3 Van Landuyt HW, Magerman K, Gordts B. The importance of the quinolones in antibacterial therapy. J Antimicrob Chemother 1990;26(Suppl D):1-6.

4 Huang ES, Stafford RS. National patterns in the treatment of urinary tract infections in women by ambulatory care physicians. Arch Intern Med 2002; 162:41-7

5 Yew WW, Piddock L, Li MS, et al. In-vitro activity of quinolones and macrolides against mycobacteria. J Antimicrob Chemother 1994;34:343-51.

6 Bozeman L, Burman W, Metchock B, et al. Fluoroquinolone susceptibility among Mycobacterium tuberculosis isolates from the United States and Canada. Clin Infect Dis 2005;40:386-91.

7 Dooley KE, Golub J, Goes FS, et al. Empiric treatment of community-acquired pneumonia with fluoroquinolones, and delays in the treatment of tuberculosis. Clin Infect Dis 2002;34:1607-12.

8 Center for Disease Control. Statistics of communicable diseases and surveillance report in Taiwan area, 2003. Taipei, Taiwan: Center for Disease Control, 2004.

9 Wang JY, Lee LN, Chou CS, et al. Performance assessment of a nested-PCR assay (the RAPID BAP-MTB) and the BD ProbeTec ET system for detection of Mycobacterium tuberculosis in clinical specimens. J Clin Microbiol 2004;42:4599-603.

10 Wang JY, Lee LN, Hsueh PR. Factors changing the manifestation of pulmonary tuberculosis. Int J Tuberc Lung Dis 2005;9:777-83.

11 Crump JA, Reller LB. Two decades of disseminated tuberculosis at a university medical center: the expanding role of mycobacterial blood culture. Clin Infect Dis 2003;37:1037-43.

12 Oursler KK, Moore RD, Bishai WR, et al. Survival of patients with pulmonary tuberculosis: clinical and molecular epidemiologic factors. Clin Infect Dis 2002;34:752-9.

13 World Health Organization. WHO report 2005: global tuberculosis control. Geneva: World Health Organization, 2005.

14 Hsu DI, Okamoto MP, Murthy R, et al. Fluoroquinolone-resistant Pseudomonas aeruginosa: risk factors for acquisition and impact on outcomes. J Antimicrob Chemother 2005;55:535-41.

15 KiaNoury D, Timpone J, Yeager H Jr. Can administration of a fluoroquinolone delay diagnosis of pulmonary tuberculosis? Int J Tuberc Lung Dis 2000;4:1092.

16 Abiad H. Does the use of fluoroquinolones for the empiric treatment of pneumonia delay initiation of treatment of tuberculosis? Clin Infect Dis 2002;35:1572-3.

17 Agarwal A. TB should be diagnosed before using a fluoroquinolone. BMJ 2003;327:164-5.

18 Golub JE, Bur S, Cronin WA, et al. Impact of empiric antibiotics and chest radiograph on delays in the diagnosis of tuberculosis. Int J Tuberc Lung Dis 2005;9:392-7.

19 Murray CK, Hospenthal DR. Treatment of multidrug resistant Acinetobacter. Curr Opin Infect Dis 2005; 18:502-6.

20 Sullivan EA, Kreiswirth BN, Palumbo L, et al. Emergence of fluoroquinoloneresistant tuberculosis in New York City. Lancet 1995;345:1 148-50.

21 Ginsburg AS, Hooper N, Parrish N, et al. Fluoroquinolone resistance in patients with newly diagnosed tuberculosis. Clin Infect Dis 2003:37:1448-52.

22 Ginsburg AS, Woolwine SC, Hooper N, et al. The rapid development of fluoroquinolone resistance in $M$ tuberculosis. N Engl J Med 2003;349:1977-8

23 Pablos-Mendez A, Sterling TR, Frieden TR. The relationship between delayed or incomplete treatment and all-cause mortality in patients with tuberculosis. JAMA 1996;276:1223-8.

24 Alwood K, Keruly J, Moore-Rice K, et al. Effectiveness of supervised, intermittent therapy for tuberculosis in HIV-infected patients. AIDS 1994;8:1103-8.

25 Wang JY, Hsueh PR, Lee LN, et al. Mycobacterium tuberculosis inducing disseminated intravascular coagulation. Thromb Haemost 2005;93:729-34.

26 Humphries MJ, Byfield SP, Darbyshire JH, et al. Deaths occurring in newly notified patients with pulmonary tuberculosis in England and Wales. Br J Dis Chest 1984;78:149-58. 
27 Joshi N, Caputo GM, Weitekamp MR, et al. Infections in patients with diabetes mellitus. N Engl J Med 1999;341:1906-12.

28 Gilboa E. How tumors escape immune destruction and what we can do about it. Cancer Immunol Immunother 1999;48:382-5.
29 Andrew OT, Schoenfeld PY, Hopewell PC, et al. Tuberculosis in patients with end-stage renal disease. Am J Med 1980;68:59-65.

30 Chandra RK, Kumari S. Nutrition and immunity: an overview. J Nutr 1994; 124:1433-5S.

\section{LUNG ALERT}

\section{Cyclophosphamide in scleroderma lung disease}

$\Delta$ Tashkin DP, Elashoff R, Clements PJ, et al. Cyclophosphamide versus placebo in scleroderma lung disease. N Engl J Med 2006;354:2655-66

round $40 \%$ of patients with systemic sclerosis will develop significant interstitial lung disease. No agent has been proved in a randomised controlled trial to be effective in this condition. This multicentre, double blind, randomised, placebo controlled trial evaluated the effectiveness of oral cyclophosphamide in patients with scleroderma who had restrictive lung function, exertional dyspnoea, and evidence of active alveolitis.

One hundred and fifty eight patients were randomised to receive cyclophosphamide (up to $2 \mathrm{mg} / \mathrm{kg} /$ day) or placebo for 1 year. There was a small but significant difference in the primary outcome measure-change in forced vital capacity (FVC) - at 12 months, favouring cyclophosphamide $(-1.0(0.92) \%$ predicted $v-2.6(0.9) \%$ predicted, $\mathrm{p}<0.03)$. This benefit was maintained after a further 12 months off study medication. There was no improvement in gas transfer but small improvements were seen in the transitional dyspnoea index and some quality of life measures. Those with more severe fibrosis at baseline had the greatest benefit in FVC.

Adverse events were more common in the cyclophosphamide group, including a significantly greater incidence of leucopenia and neutropenia. The potential longer term associations with malignancy were not evaluated within this 2 year period.

This is the first placebo controlled study to demonstrate a benefit of treatment for scleroderma related interstitial lung disease. However, these benefits were small and need to be weighed against the risk of adverse side effects.

Specialist Registrar, Royal United Hospital, Bath, UK; henrysteer@hotmail.com

\section{LUNG ALERT}

\section{No need to stay in hospital after antibiotic switch in pneumonia}

$\Delta$ Nathan RV, Rhew DC, Murray C, et al. In-hospital observation after antibiotic switch in pneumonia: a national evaluation. Am J Med 2006;119:512-8

$\mathrm{T}$ his was a multicentre retrospective analysis during one of two 6 month periods in 19989 which assessed the benefit of in-hospital observation after the switch from intravenous to oral antibiotics in patients with community acquired pneumonia (CAP) aged at least 65 years. The US Medicare National Pneumonia Project database was used to create a sample population from 4341 hospitals in all 50 states of the US and the District of Columbia. 5248 patients fulfilled the eligibility criteria and were divided into two groups (2536 "not observed" and 2712 "observed for 1 day").

There was no significant difference between the groups in 14 day hospital re-admission rate $(7.8 \%$ in the "not observed" $v 7.2 \%$ in the "observed for 1 day" group, $\mathrm{p}=0.367$ ) or 30 day mortality $(5.1 \%$ in the "not observed" $v 4.4 \%$ in the "observed for 1 day" group, $\mathrm{p}=0.258$ ). There were substantial economic implications of early discharge, with projected annual savings of up to $\$ 27.1$ million. There were a few limitations to the study; the number of days of observation after the switch was based on calendar days and not hours, and those patients who were observed for 1 day to address active medical or social reasons were also included in the "observed for 1 day" group.

The authors conclude that low risk patients with CAP can be safely discharged soon after changing from intravenous to oral antibiotics, although there was a non-significant trend to lower 30 day mortality in those observed. The potential benefits of such an approach are shorter hospital stays and, perhaps, greater patient satisfaction. However, a randomised controlled trial is required to confirm the results of this study. 